\title{
X-RAY TRANSIENTS - RESULTS OF ROSAT OBSERVATIONS
}

\author{
J. TRÜMPER, K. DENNERL AND J. ENGLHAUSER \\ Max-Planck-Institut für extraterrestrische Physik \\ $D-85748$ Garching, Germany
}

\section{Observations of $\mathrm{X}$-ray variability with ROSAT}

The main strengths of ROSAT [1] are its high quality imaging of extended sources and its large sensitivity for the detection of point sources. In the all-sky survey the diffuse $\mathrm{X}$-ray emission was mapped with a resolution of $12 \operatorname{arcmin}[2]$. The number of point sources detected in the survey is $\approx 80000$ [3]. In addition more than 80000 sources have been found in the $\approx 6500$ fields observed with the PSPC and HRI until now in the course of the pointed programme. A wealth of information about source variabilities has been extracted both from the all-sky survey and from the pointed observations, as well as from a combination of both. The outcome of correlation studies on source variabilities has been given by Voges and Boller 1997, cf. Fig. 1 [4]. In this article we can mention only a few scientific highlights:

- the discovery of highly variable supersoft sources [5] and their identification with binary white dwarfs showing unstable nuclear burning at the surface.

- the discovery of a lower bound of the X-ray surface flux from stellar coronae in G- and K-stars which is identical to the solar coronal flux observed in coronal holes [6].

- the discovery of rapid X-ray variability in Narrow Line Seyfert Galaxies which can be interpreted in terms of relativistic beaming occurring in the inner part of an accretion disk around a black hole [7].

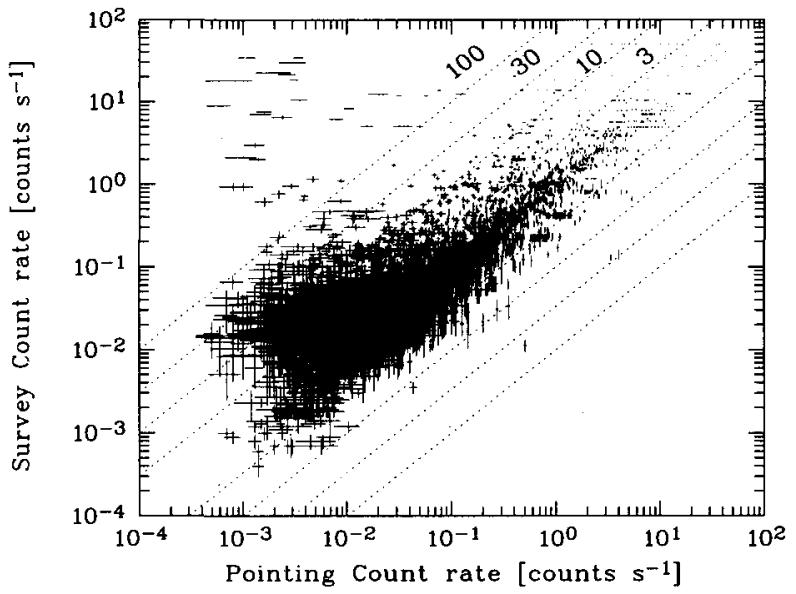

Fig. 1. Count rate within the RASS observations versus count rate within pointed ROSAT observations on sources which are spatially coincident. The factor of variability is marked by the dashed lines (four values are shown indicating factors of variability of $3,10,30$ and 100 , respectively). Objects in the upper left side of the diagram are brighter during the survey observations with respect to the subsequent pointed observations. The opposite holds for objects in the lower right side of the diagram.

Another highlight was the unexpected discovery of X-ray emission from Comets $[8,9]$ which represent a very old class of "transients" in astronomy. Due to the ROSAT observations they have now become high energy transients and therefore fit into scope of this joint discussion. 


\section{X-ray emission from Comets}

ROSAT observations of comet C/1996 B2 (Hyakutake), triggered by a very favorable observing geometry, led to the discovery of unexpectedly bright $\mathrm{X}$-ray emission [8]. The emission came from a crescent-shaped region which was symmetric to the sun-comet line and offset sunward by $\approx 18000 \mathrm{~km}$. It was variable on time scales of hours, which is shorter than the 6.2 hour rotation period of the nucleus. The total $\mathrm{X}$-ray luminosity varied between $4 \cdot 10^{15} \mathrm{ergs} \mathrm{s}^{-1}$ and $16 \cdot 10^{15} \mathrm{ergs} \mathrm{s}^{-1}$. A comparison of the XUV and X-ray fluxes indicated a soft X-ray spectrum, with a typical energy $E<1 \mathrm{keV}$.

The challenge of explaining the origin of the $\mathrm{X}$-ray emission triggered many activities on the theoretical side: comets are cool objects, and the processes which had been previously observed to take place in the coma operate at moderate temperatures. Strong $\mathrm{X}$-ray emission was not a natural consequence of the current picture of what was thought to be going on in a cometary coma of gas and dust. Attempts to explain it by scattering of solar X-rays in gas or normal-sized dust grains failed to reach the required intensity. While very small dust particles $(\approx 30 \AA$ size $)$ are more efficient in scattering soft $\mathrm{X}$-rays [10], the temporal variations of the Hyakutake $\mathrm{X}$-ray flux were considerably different from the $2-12 \mathrm{keV}$ solar X-ray flux, monitored simultaneously with the GOES- 8 satellite. The suggestion that the X-ray emission was caused by collisions between cometary and interplanetary dust particles [11] failed to reproduce the observed intensity and morphology. Interactions with the solar wind, either with the protons, heavier ions, energetic electrons, or its magnetic field, were considered as an alternative cause for the $\mathrm{X}$-ray emission, although no mechanism was found at these early stages which explained the observed properties in a straighforward way. In view of these difficulties there was also the possibility that Hyakutake had been observed in an unusual ' $\mathrm{X}$-ray bright' state, and that such intense $\mathrm{X}$-ray emission would not be a general property of comets.

On the observational side, the fact that one comet was now known to be capable of emitting $\mathrm{X}$-rays, triggered a systematic search in ROSAT archival data: in 1990/91 ROSAT had performed the first survey of the whole sky with an imaging $X$-ray telescope [12]. By chance comets might have crossed the field of view and left traces in the data. For this search, all of the comets which were known to have passed their perihelion between 1985 and 1997 were selected [13]. Of these 214 comets, 25 turned out to have crossed the ROSAT field of view when they were at heliocentric distances of less than three astronomical units (AU). To obtain the maximum detection efficiency, the search was performed in the rest frame of each comet. This was possible since the arrival time and the detector coordinates of each detected X-ray photon were recorded. Thus, the apparent proper motion of the comet as seen from the satellite could be tracked, given the the satellite position together with the comet ephemeris. The search resulted in the discovery of X-ray emission from four comets, seen on seven occasions at different states [9]. These comets are C/1990 K1 (Levy), C/1990 N1 (Tsuchiya-Kiuchi), 45P/Honda-Mrkos-Pajdušáková and C/1991 A2 (Arai). They were optically 300 to 30000 times fainter than Hyakutake. In the case of comet C/1991 A2 (Arai), the $\mathrm{X}$-ray observations took place six weeks before the comet was (optically) discovered. All of the comets which were observed at heliocentric distances of less $2 \mathrm{AU}$ and with total visual magnitude $m_{1}<12$ mag were detected in X-rays (Fig. 2). Thus, X-ray emission appears to be a direct product of cometary activity.

The all-sky survey was performed with a Position Sensitive Proportional Counter (PSPC [14]) in the focal plane of the $\mathrm{X}$-ray telescope. The unlimited field of view provided by the all-sky survey together with the low instrumental background of the PSPC and its high sensitivity to soft X-rays makes it well suited for studying faint extended objects with soft spectra. Most of the $\mathrm{x}$-ray images show emissions which are roughly spherically symmetric or elongated perpendicular to the sun comet line, with the brightest peak usually being offset sunward by some $10^{4} \mathrm{~km}$, reminiscent of a bow shock structure. In the case of comet Levy, it is possible to trace the $\mathrm{X}$-ray emission out to a radial distance of at least $2 \cdot 10^{6} \mathrm{~km}$, while in none of the images evidence for X-ray emission from the gas or dust tail is found. There is no clear dependence of the $\mathrm{X}$-ray morphology on the velocity vector of the comet. The PSPC provides an energy resolution of $\Delta E / E=0.43 \cdot \sqrt{0.93 / E[\mathrm{keV}]}$ over a bandpass of $0.1-2.4 \mathrm{keV}$. Thus, spectroscopic studies of cometary $\mathrm{X}$-ray emission are possible 


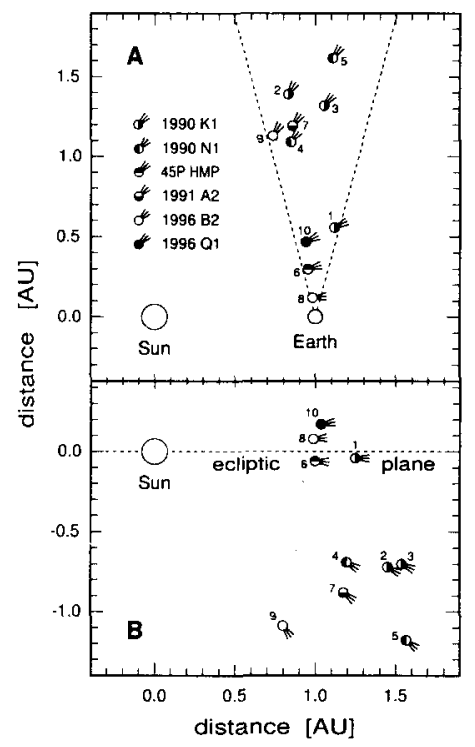

Fig. 2. Position of the comets detected in X-rays with respect to the sun and Earth $(\mathbf{A})$ and the ecliptic plane (B). The dashed lines in $(A)$ enclose the ROSAT observing window. Different symbols indicate different comets; numbers identify the individual observations. \#1-\#7 were serendipitous observations during the all-sky survey in 1990/91, while \#8-\#10 were dedicated comet observations in 1996.

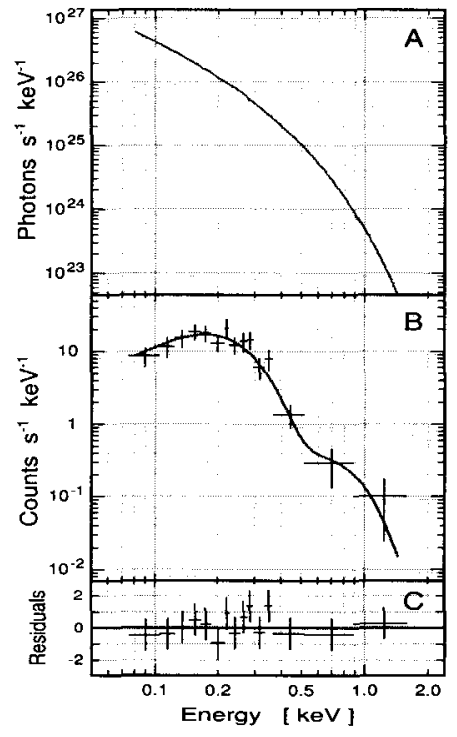

Fig. 3. X-ray spectrum of comet C/1990 K1 (Levy): (A) Spectral model: photon spectrum of thermal bremsstrahlung emission with $k T=$ $0.23 \mathrm{keV}$. (B) Observed PSPC pulse height distribution (crosses with $1 \sigma$ error bars) with pulse height distribution resulting from the model spectrum. (C) Residual deviations of the observed data from the model. The confidence of the fit is $89 \%$.

with unprecedented quality. In the seven observations the emission is very soft: about $95 \%$ of the detected photons are at $E<0.4 \mathrm{keV}$. This corresponds to $83 \%$ of the energy flux in the total PSPC bandpass. A good empirical description of all the cometary $\mathrm{X}$-ray spectra, within the available spectral resolution, is provided by thermal bremsstrahlung emission with $k T=0.2 \mathrm{keV}$ (Fig. 3). Comparison of the $\mathrm{X}$-ray luminosity $L_{\mathbf{x}}$ with the optical luminosity $L_{\mathrm{opt}}$ shows that $L_{\mathrm{x}}$ is about proportional to $L_{\mathrm{opt}}$, with $L_{\mathrm{x}} \approx 10^{-5} \cdot L_{\mathrm{opt}}$, although there are differences in the $L_{\mathrm{x}} / L_{\mathrm{opt}}$ ratio between different comets. These differences are not related to the solar activity cycle: comets in the all-sky survey were observed at the solar maximum, while the recent comet observations took place during solar minimum. The differences may, however, be related to the ratio between the dust and gas in the coma: there is evidence that $L_{\mathrm{x}}$ is related to the gas content rather than the dust content in the coma, while $L_{\text {opt }}$ is known to be controlled mainly by cometary dust.

The fact that the $\mathrm{X}$-ray emission can be traced over several $10^{6} \mathrm{~km}$, favors the gas coma as the target for the interaction, because only cometary gas, in particular hydrogen, is known to extend over such distances. This is further supported by the positive correlation of the $L_{\mathbf{x}} / L_{\text {opt }}$ ratio with the gas/dust ratio between different comets. Since no correlation between the $\mathrm{X}$-ray intensity of a comet and the solar X-ray flux is observed, it is unlikely that solar $\mathrm{X}$-ray radiation is primarily responsible for the $\mathrm{X}$-ray emission. Another argument against solar $\mathrm{X}$-rays is the fact that no $\mathrm{X}$-ray emission is observed from the tail. All observational evidence favors the idea that the $\mathrm{X}$-ray emission is caused by an interaction between the solar wind and the gas in the coma.

The most straightforward process for this interaction is charge exchange between highly charged heavy ions in the solar wind and cometary neutrals [15]. Due to the high cross-section of this process $\left(>10^{-15} \mathrm{~cm}^{-2}\right)$, the energy stored in the heavy ions is consumed before they reach the innermost part of the coma. This explains both the observed morphology and high $\mathrm{X}$-ray luminosity. Charge exchange produces an $\mathrm{X}$-ray spectrum with so many $(>100)$ emission lines that for an instrument 
with moderate spectral resolution, this spectrum would appear like a continuum. The spectral shape of this continuum can be well approximated by $0.2 \mathrm{keV}$ thermal bremsstrahlung emission [16], in agreement with the observed ROSAT PSPC spectra. The charge exchange process has an interesting consequence: while self-absorption of $\mathrm{X}$-rays within the coma is negligible, the inner part of the coma is collisionally thick with respect to charge transfer, as long as the gas production rate exceeds $10^{26}$ molecules $\mathrm{s}^{-1}$ (i.e. even for only moderately active comets). Thus, the peak X-ray surface flux should scale with the heavy ion flux in the solar wind, independent of individual comet properties. This is in agreement with the ROSAT observations, as the derived peak surface fluxes are consistent with a $r^{-2}$ dependence of heliocentric distance $r$. It is very likely that the $\mathrm{X}$-ray variability of comets is caused by a similar variability of the heavy ion flux, although this has still to be proven in detail.

All the observational evidence is in favor of charge exchange between highly charged heavy ions in the solar wind and cometary neutrals as the dominant process for the $\mathrm{X}$-ray emission of comets. It might thus be possible to use comets as natural space probes for monitoring the temporal and spatial fluctuations of these solar wind ions, at various positions in the inner solar system.

\section{References}

1. Trümper, J., Adv. Space Res. 2.4, 241 (1983)

2. Snowden, S., Egger, R., Freyberg, M.J., McCammon, D., Plucinsky, P.P., Sanders, W.T., Schmitt, J.H.M.M., Trümper, J., Voges, W., ApJ 485, 125 (1997)

3. Voges W., Boller, Th., Dennerl, K., Englhauser, J., Gruber, R., Haberl, F., Paul, J., Pietsch, W., Trümper, J., Zimmermann, H.U., MPE Report 263 (1996)

4. Voges W. and Boller Th., Astron. Nachr. 319 (1998)

5. Trümper, J., Hasinger, G., Aschenbach B., Bräuninger, H., Briel, U.G., Burkert, W., Fink, H., Pfeffermann, E., Pietsch, W., Predehl, P., Schmitt, J.H.M.M., Voges, W., Zimmermann, U., Beuermann, K., Nature 349, 579-583 (1991)

6. Schmitt, J.H.M.M., Astron. Astrophys. 318, 215 (1997)

7. Boller, Th., Brandt, W.N., Fabian, A.C., and Fink, H.H., Mon. Not. R. Astron. Soc. 289, 393-405 (1997)

8. Lisse, C.M., Dennerl, K., Englhauser, J., Harden, M., Marshall, F.E., Mumma, M.J., Petre, R., Pye, J.P., Ricketts, M.J., Schmitt, J., Trümper, J., West, R.G., Science, 274, 205-209 (1996)

9. Dennerl, K., Englhauser, J., Trümper, J., Science, 277, 1625-1630 (1997)

10. Wickramasinghe, N. C. and Hoyle, F., Astrophysics and Space Science 239, 121-123 (1996)

11. Ibadov, S., Icarus 86, 283-288 (1990)

12. Voges, W., Proc. of Satellite Symp. 3: Space Sciences with particular emphasis on High-Energy Astrophysics, from the 'International Space Year' Conference held in Munich, Germany, 30 March - 4 April 1992 (ESA ISY-3, July 1992, p. 9) (1992)

13. Catalogue of Cometary Orbits, 11th Edition 1996, Central Bureau for Astronomical Telegrams / Minor Planet Center (1996)

14. Pfeffermann, E., Briel, U.G., Hippmann, H., Kettenring, G., Metzner, G., Predehl, P., Reger, G., Stephan, K.-H., Zombeck, M.V., Chappell, J., Murray, S.S., Proc. SPIE 733, 519 (1986)

15. Cravens, T.E., Geophysical Research Letters 24.1, $105-108$ (1997)

16. Wegmann, R., Schmidt, H.U., Lisse, C.M., Dennerl, K., Englhauser, J., Planetary and Space Science, in press (1997) 